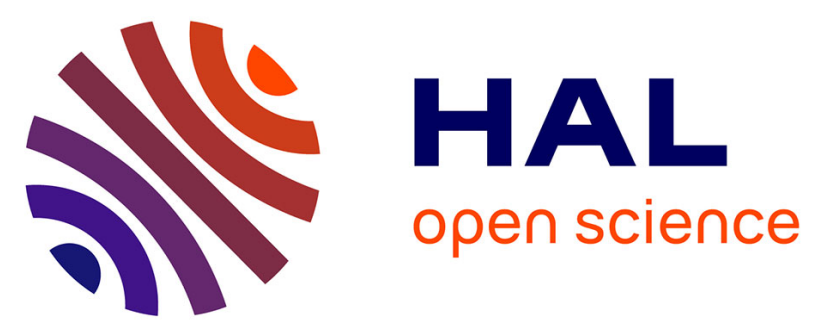

\title{
Status of PRESS-MAG-O: The experimental apparatus to probe materials and phenomena under extreme conditions at Frascati
}

Daniele Di Gioacchino, Augusto Marcelli, Mariangela Cestelli Guidi, Massimo Piccinini, Alessandro Puri, Paolo Postorino, Emanuele Pace, Antonio de Sio, Lisa Gambicorti

\section{To cite this version:}

Daniele Di Gioacchino, Augusto Marcelli, Mariangela Cestelli Guidi, Massimo Piccinini, Alessandro Puri, et al.. Status of PRESS-MAG-O: The experimental apparatus to probe materials and phenomena under extreme conditions at Frascati. Journal of Physics and Chemistry of Solids, 2010, 71 (8), pp.1042. 10.1016/j.jpcs.2010.03.003 . hal-00661918

\section{HAL Id: hal-00661918 https://hal.science/hal-00661918}

Submitted on 21 Jan 2012

HAL is a multi-disciplinary open access archive for the deposit and dissemination of scientific research documents, whether they are published or not. The documents may come from teaching and research institutions in France or abroad, or from public or private research centers.
L'archive ouverte pluridisciplinaire HAL, est destinée au dépôt et à la diffusion de documents scientifiques de niveau recherche, publiés ou non, émanant des établissements d'enseignement et de recherche français ou étrangers, des laboratoires publics ou privés. 


\section{Author's Accepted Manuscript}

Status of PRESS-MAG-O: The experimental apparatus to probe materials and phenomena under extreme conditions at Frascati

Daniele Di Gioacchino, Augusto Marcelli, Mariangela Cestelli Guidi, Massimo Piccinini, Alessandro Puri, Paolo Postorino, Emanuele Pace, Antonio De Sio, Lisa Gambicorti

PII: S0022-3697(10)00049-1

DOI: doi:10.1016/j.jpcs.2010.03.003

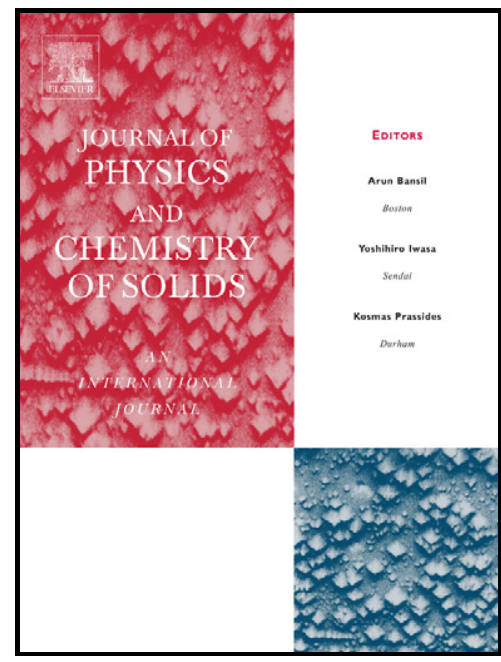

www.elsevier.com/locate/jpcs

Reference: PCS 6112

To appear in: $\quad$ Journal of Physics and Chemistry of Solids

Cite this article as: Daniele Di Gioacchino, Augusto Marcelli, Mariangela Cestelli Guidi, Massimo Piccinini, Alessandro Puri, Paolo Postorino, Emanuele Pace, Antonio De Sio and Lisa Gambicorti, Status of PRESS-MAG-O: The experimental apparatus to probe materials and phenomena under extreme conditions at Frascati, Journal of Physics and Chemistry of Solids, doi:10.1016/j.jpcs.2010.03.003

This is a PDF file of an unedited manuscript that has been accepted for publication. As a service to our customers we are providing this early version of the manuscript. The manuscript will undergo copyediting, typesetting, and review of the resulting galley proof before it is published in its final citable form. Please note that during the production process errors may be discovered which could affect the content, and all legal disclaimers that apply to the journal pertain. 


\title{
Status of PRESS-MAG-O: the experimental apparatus to probe materials and phenomena under extreme conditions at Frascati
}

\author{
Daniele Di Gioacchino ${ }^{\mathrm{a}}$, Augusto Marcelli ${ }^{\mathrm{a}}$, Mariangela Cestelli Guidi ${ }^{\mathrm{a}}$, Massimo Piccinini ${ }^{\mathrm{a}}$, \\ Alessandro Puria ${ }^{\mathrm{a} b}$, Paolo Postorino ${ }^{\mathrm{c}}$, Emanuele Pace ${ }^{\mathrm{d}}$, Antonio De Sio ${ }^{\mathrm{d}}$, Lisa Gambicorti ${ }^{\mathrm{e}}$ \\ ${ }^{a}$ INFN - LNF, Via E. Fermi, 40 - 00044 Frascati (Rome), Italy \\ bUniversity 'La Sapienza', Dipartimento di Fisica, P.le A. Moro, 4 - 00100 Rome, Italy \\ 'INFM-CNR - Coherentia, University 'La Sapienza', P.le A. Moro, 4 - 00100 Rome, Italy \\ ${ }^{\mathrm{d}}$ INFN, Dept. of Astronomy and Space Science, University of Firenze, L.go E. Fermi, 2 \\ 50125 Firenze, Italy \\ ${ }^{\mathrm{e}}$ INFN, CNR-INOA, National Institute of Applied Optics, L.go E. Fermi, 6 - 50125 Firenze, Italy
}

\begin{abstract}
In this contribution we up-to-date the status of the PRESS-MAG-O device, a new instrument under commissioning at the INFN designed to perform magnetic and spectroscopic experiments on samples under extreme conditions. The system has been designed to work at SINBAD, the IR synchrotron radiation beamline operational at $D A \Phi N E$. The instrument, that is the result of a significant R\&D, will allow performing concurrent high harmonic ac magnetic susceptibility measurements and magneto-optic experiments on a sample under high pressure, with a variable DC magnetic field in a wide temperature range. The vacuum vessel has been designed with four crossing windows to allow optical measurements in the transmission geometry on the sample loaded inside a Diamond Anvil Cell. A new superconducting miniaturized micro-SQUID gradiometer has been also developed to detect the low magnetic signal of the sample and a customized optical system has been also designed to perform IR synchrotron radiation experiments.
\end{abstract}

Phase transition, High Pressure, High harmonic magnetic ac susceptibility measurements, IR spectroscopy.

\section{INTRODUCTION}

PRESS-MAG-O is an original device designed to investigate materials under extreme conditions, i.e., as a function of both pressure and DC magnetic field and in a wide temperature range [1]. The apparatus has been completely developed at the INFN as the result of a project funded by the INFN [2]. Materials like ferroelectrics or superconducting systems, magnetic transitions and new condensed matter phases can be investigated with this device that permits concurrent magnetic and optical experiments. A FTIR spectroscopic analysis in a wide IR domain with magnetic experiments represents a new strategic approach combining information of the phonon behavior with linear and nonlinear electron dynamic transport processes.

\section{THE PRESS-MAG-O SYSTEM}

The apparatus is the result of a significant R\&D in different areas. Several technical advancements guaranteed the construction of the cryostat and its internal components thanks also to the availability of new materials and a high level technological processes.

\subsection{The cryostat}

The cryostat showed in Fig. 1-left is a compact device made by an a-magnetic stainless steel vacuum vessel with two shields at the liquid nitrogen temperature and four access ports for optical experiments.

Inside we have an internal liquid helium reservoir hosting an $8 \mathrm{~T}$ superconducting split coil magnet (see Fig. 1-right) with four radial pipes, a vertical port to insert the sample holder and a bottom cold finger. The cryostat allows concurrent magneto-optics experiments thanks to two optical windows transmitting light from the visible down to the far-IR range. An important component of this device is the 'sample-insert' device designed to host the Diamond Anvil Cell (DAC) and a miniaturized magnetometer with sensitivity in the fT range [2].

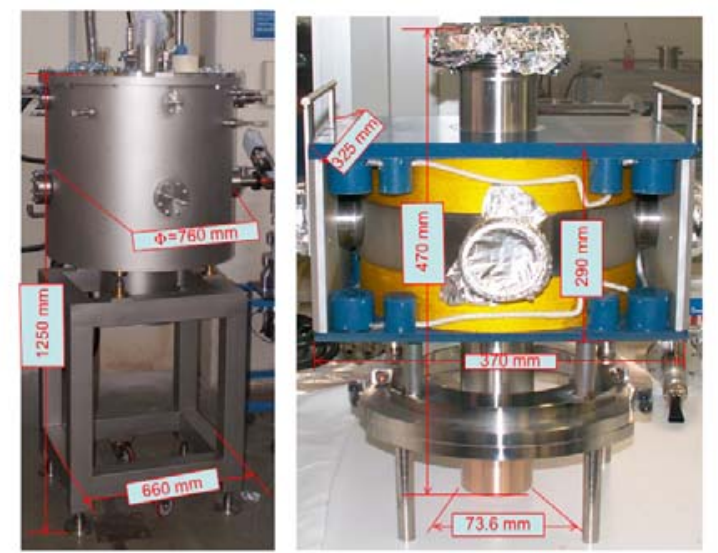

Fig. 1. Photographs of the cryostat (left) and of the $8 \mathrm{~T}$ superconducting magnet with its four radial access ports (right).

\subsection{The sample-insert}

The sample insert showed disassembled in the left panel of Fig. 2 is the heart of the PRESS-MAG-O system. It can be divided in two sections, each containing important components. A load-lock system installed on the vacuum flange on the upper cover of the cryostat allows the motion of the sample holder, still maintaining the cryostat under vacuum. The upper part of the holder (Fig. 2 - left) with its $\mathrm{X}-\mathrm{Y}-\Theta$ control and micrometer accuracy will be used to align the light inside a DAC loading the sample inside a 
gasket with internal diameter of $\sim 400 \mu \mathrm{m}$. In the bottom of the holder we have the $\mathrm{Cu}-\mathrm{Be}$ sample-holder shaped with some breaks to contain (figure 2 left): 1) the DAC with proper $\mathrm{Cu}-\mathrm{Be}$ springs to guarantee the cold contact via an 'external fixed element' with the cold finger ; 2) an excitation coil slider to fit the micro-gradiometer inside the DAC. The SQUID amplifier, being in thermal contact with the cold finger of the cryostat, fits nicely inside the 'fixed external element'

After technical evaluations and tests with an ac magnetic field produced by excitation coils, the original $\mathrm{Cu}-\mathrm{Be}$ sample-holder was redesigned in sapphire, an optimal thermal conductor but electrical insulator. The new DAC holder allows minimizing the 'Foucault current' losses inside the bulk $\mathrm{Cu}$-Be material still maintaining a good thermal contact (see the right panel of Fig. 2). In fact, although it was designed with a particular shape, due to Foucault losses the previous system dissipated about $80 \%$ of magnetic signal.

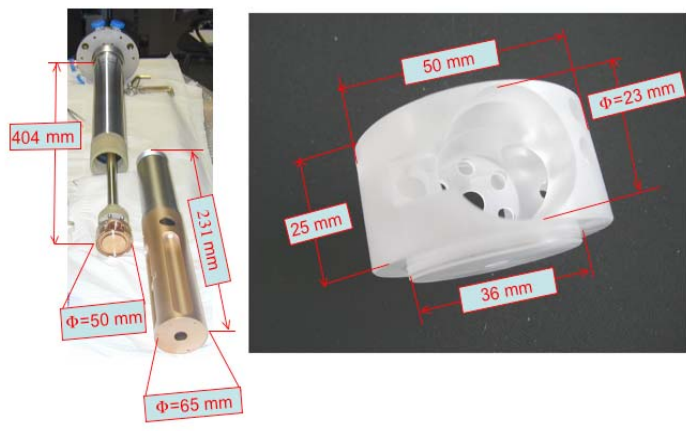

Fig. 2. Photographs of the disassembled PRESS-MAG-O insert with its main components (left) and the sapphire sample-holder (right).

With this new original solution we measured intensity values of a sinusoidal field from 1 to 20 Gauss on the position of the sample at the center of the DAC cell.

As showed in Fig. 3, this result has been obtained applying

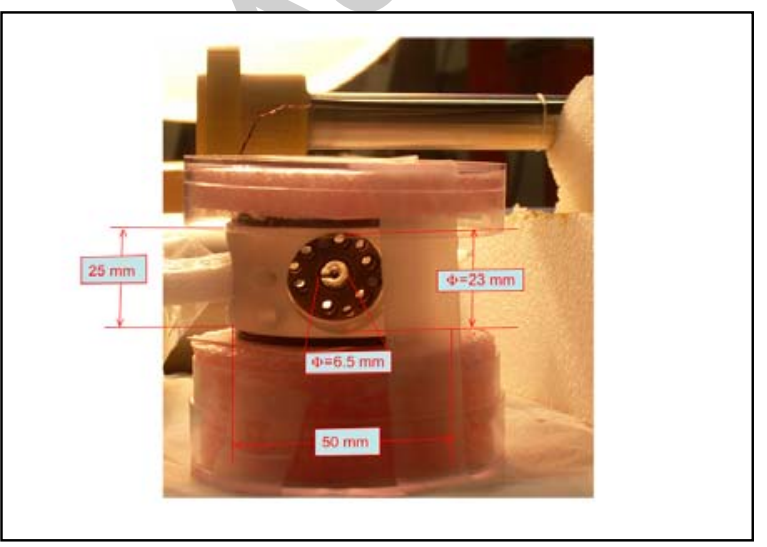

Fig. 3. Photograph of the Hall probe placed inside the DAC and loaded in the sapphire sample-holder with the two external split excitation coils.

an ac magnetic field with the split excitation coils and measuring the field with a Hall probe placed inside the DAC and loaded in the sapphire sample-holder.

Owing to an ac generator working in the range 200-5500 $\mathrm{mV}$ at $17-1070 \mathrm{~Hz}$, measurements were possible. Scope measurements demonstrated also that the signal is not distorted. A linear trend of the magnetic field inside the DAC has been observed when the voltage is in the range 1-20 Gauss for all frequencies (see Fig. 4). However, for $\mathrm{V}>5000 \mathrm{mV}$ and at frequencies $\geq 707 \mathrm{~Hz}$ the generator saturates. Deviations from linearity have been observed limiting the maximum applicable field.

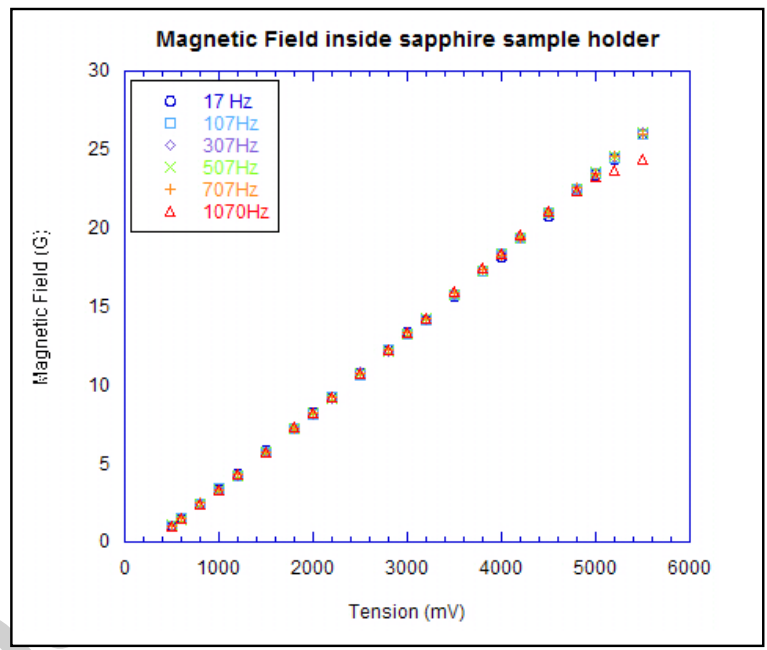

Fig. 4. Plot of the magnetic field measured inside the sapphire sampleholder vs. applied voltage.

\subsection{The DAC and the gradiometer}

For the magnetic experiments we designed and manufactured a small DAC cell made in $\mathrm{Cu}$-Be. In more details it is composed by two $\mathrm{Cu}-\mathrm{Be}$ disks, two brilliant cut diamonds of type IIa necessary to perform experiments in the IR range [3] and two SiC- $\delta$ cylinders with a $400 \mu \mathrm{m}$ conic hole to push diamond anvils. The micro-gradiometer sensor showed in Fig. 5 is made by two photolithography

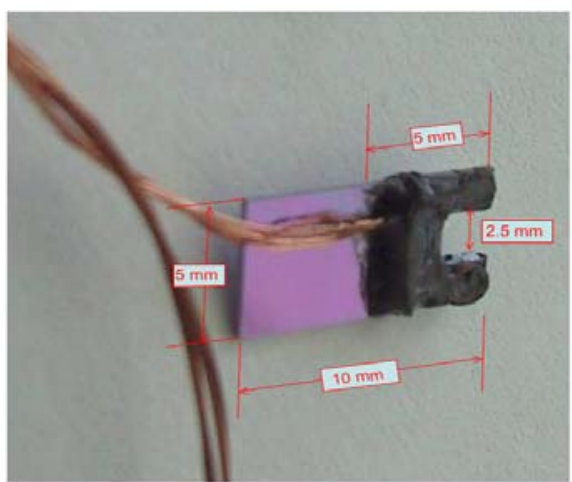

Fig. 5. Photograph of the micro-pick-up coils to be inserted inside the DAC cell.

coils manufactured on a mono-crystal silicon wafer with size and layout in order to match the small space inside the 
DAC, and as near as possible to the gasket were sample is loaded. This original pick-up magnetic system design has been well described in Ref. 2.

An innovative DAC is also under study and development. This is based on the idea of improving both the heat transfer to the sample and the related measurement of the temperature. The major problem to solve is to heat the sample uniformly at a desired temperature and therefore to measure precisely that temperature.

To achieve this innovative result, we will apply directly on the diamond anvil surface a micro-heater and a microthermometer. Taking advantage from the very high thermal conductivity of diamond, this heater and sensor will be placed on the top surface that is larger, thus allowing the arrangement of both.

Another possible solution to heating uniformly the sample is to use moissanite, which is silicon carbide. [4] This material can be also applied in high pressure experiments as anvils [5]. In our case, a moissanite thick layer can be used to arrange the heater and the sensor and then coupled to the diamond anvil. This option, even introducing an interface between diamond and moissanite, would allow a simpler setup arrangement by exploiting the high thermal conductivity of both moissanite and diamond.

Presently, we are performing technological trials on a diamond anvil having a heater and a thermometer on its top circular surface having a 3-mm diameter. The size of both the devices is of the order of $1 \mathrm{~mm}$. These preliminary tests will provide us information about the optimal parameters to fabricate practical devices on diamond. We are planning to perform similar tests on moissanite disks in order to compare the two technologies and to verify the feasibility of coupling between the two materials.

The main issue will be the electrical behavior of both the devices with temperature. In fact, the technology we are applying has been already developed for devices at room temperature. Magneto-optical properties of the samples in the PRESS-MAG-O instrument will be measured at high pressure and low temperature. Thus, the performances of the devices at low temperature have to be tested.

\subsection{The optical system}

The PRESS-MAG-O instrument has been designed to perform optical experiments such as FTIR spectroscopy in transmission or reflection modes and Raman spectroscopy. SINBAD (Synchrotron Infrared Beamline At DAФNE), the first Italian IR beamline that exploited the great advantages of the synchrotron radiation sources in the IR domain, is operational at the INFN, National Laboratories of Frascati, since 2001 [6]. A brilliant IR SR source is ideal to perform high-pressure investigations on small samples inside a DAC and this is particularly true at DAФNE (Double Annular $\Phi$-factory for Nice Experiments), the Frascati electron-positron collider working in topping up mode at an energy of $0.51 \mathrm{GeV}$ per beam with a maximum beam current $>2 \mathrm{~A}$.

SINBAD has been designed to work at IR wavelengths from about 10 up to $10000 \mathrm{~cm}^{-1}$ and operates with a customized BRUKER Equinox 55 interferometer working in vacuum coupled to a BRUKER Hyperion 3000 microscope. Different experiments have been performed using DACs, in particular those on $\mathrm{La}_{1-\mathrm{x}} \mathrm{Ca}_{\mathrm{x}} \mathrm{MnO}_{3-\mathrm{x}}$ manganite samples at pressures up to $10 \mathrm{GPa}$ using the IR synchrotron radiation emission [7].

In more detail, the collimated beam coming out from the interferometer and entering the PRESS-MAG-O setup will be focused inside the DAC on the loaded sample by using one of the four lateral ports equipped by optical transmitting windows. Different windows can be installed: a CVD wedged diamond window is an option to cover the widest IR range down to the far-IR domain [3].

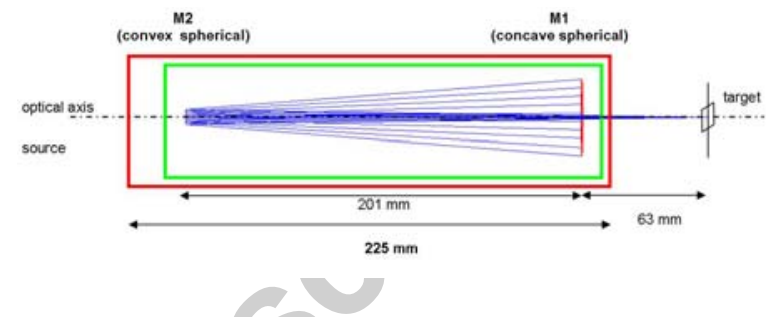

Fig. 6. Layout ray tracing of the Cassegrain telescope designed for the optical line of the cryostat.

A Cassegrain concentrator has been designed to fit inside the dimension of the apparatus in order to focus the synchrotron radiation in a small-size spot (the diameter of the Airy disk is $\sim 200 \mu \mathrm{m}$ at the shortest wavelength) inside the diamond anvil cell. The ray-tracing analysis shows in Fig. 6 as focusing is achieved by a Cassegrain telescope with $4 \%$ central obstruction, working in the midIR and far-IR range down to $\sim 100 \mathrm{~cm}^{-1}$. The mechanical layout is shown in Fig. 7.

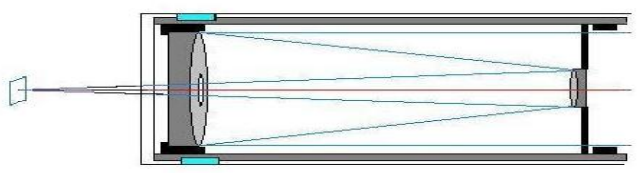

Fig. 7. Mechanical layout of the Cassegrain telescope designed for the optical line of the cryostat.

The optical system is diffraction limited in the wavelength range of interest and the spot diagram is inside the Airy Disk. Fig. 8 shows the spot diagram obtained at $2.5 \mu \mathrm{m}$ collected in a square of $200 \mu \mathrm{m}$ side.

The quality of the image is also represented by the Strehl Ratio of the Point Spread Function (PSF). It results 100\%, at shortest wavelength, as showed in Fig. 9. For IR transmission experiments, the beam will exit through a second window placed on the opposite lateral port and then focused to the detector position by an almost symmetrical Cassegrain lens.

For Raman experiments with a DAC cell [8] or measurements performed in the reflection geometry, as in 


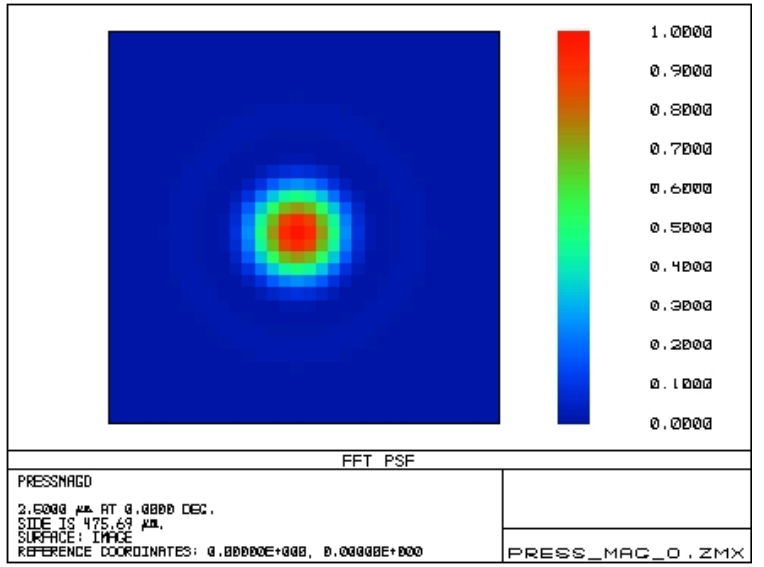

Fig. 8. Spot diagram at $2.5 \mu \mathrm{m}$. The spot is collected inside the $200 \mu \mathrm{m}$ Airy disk. The white square represents a $200 \mu \mathrm{m}$ detector pixel.

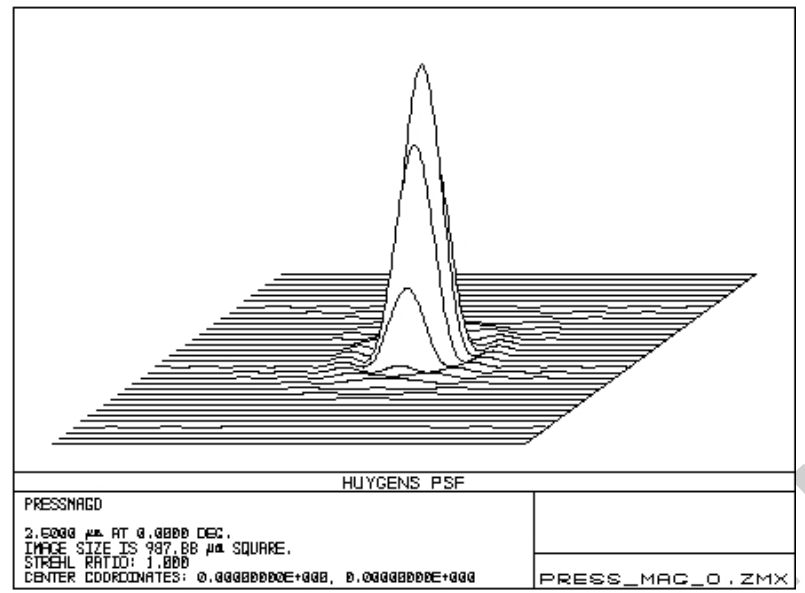

Fig. 9. PSF at shortest wavelength in PRESSMAGO range of interest. The Strehl Ratio results $100 \%$ and the optical system is diffraction limited.

a microscope optical layout, the beam will be collected by half of the Cassegrain optics used for the incoming beam in the transmission geometry.

\section{ACKNOWLEDGMENTS}

A special thank is due to F. Tabacchioni (INAF) and the entire technical staff of the DA $\sqrt{ } \mathrm{NE}-$ Light laboratory for their continuous technical support.

\section{REFERENCES}

[1] V.V. Strunzhin, R.J. Hemley and H. Mao, J. Phys: Cond. Mat. 16 (2004) S1071-S1086

[2] D. Di Gioacchino, P. Tripodi, A. Marcelli, M. Cestelli Guidi, M. Piccinini, P. Postorino, D. Di Castro, E. Arcangeletti, J. Phys. Chem. Solid 69 (2008) 2213-2216.

[3] P. Dore, A. Nucara, D. Cannavo', G. De Marzi, P. Calvani, A. Marcelli, R.S. Sussmann, A.J. Whitehead, C.N. Dodge, A.J. Krehan and H.J. Peters, Appl. Opt. 37, (1998) 5731

[4] Ji-an Xu and Ho-kwang Mao, Science 290, 783 (2000)

[5] Zhenxian Liu, Jian Xu, Henry P. Scott, Quentin Williams, Hokwang Mao and Russell J. Hemley, Rev. Sci. Instr. 75, 5026, 2004
[6] M. Cestelli Guidi, M. Piccinini, A. Marcelli, A. Nucara, P. Calvani and E. Burattini, J. Opt. Soc. Amer. A 22 (2005) 2810.

[7] A. Sacchetti, M. Cestelli Guidi, E. Arcangeletti, A. Nucara, P. Calvani, M. Piccinini, A. Marcelli and P. Postorino, Phys. Rev. Lett. 96 (2006) 035503.

[8] E. Arcangeletti, L. Baldassarre, D. Di Castro, S. Lupi, L. Malavasi, C. Marini, A. Perucchi and P. Postorino, Phys. Rev. Lett. 98, 196406 (2007) 